\title{
Two stages of motion adaptation in human visual system
}

\author{
S. A. MAHMUD \\ University of Dhaka, Dhaka, Bangladesh
}

(Judith P. Goggin, Sponsor)

\begin{abstract}
In two experiments using positive adaptation sequences followed by reverse negative ones, the presence of two separate movement aftereffects was studied. In Experiment 1 subjects were adapted binocularly, whereas in Experiment 2 monocular adaptation was employed. It was found that the movement aftereffect, like the negative afterimage, displays quite separate adaptation mechanisms-a long-term and a short-term mechanism. This indicates the existence of two distinct mechanisms of adaptation, connected in some way but occurring at different stages of visual adaptation.
\end{abstract}

Although the short-term movement aftereffect has been observed for centuries, Masland (1969) was the first to generate a relatively long-term modification of vision. He elicited a motion aftereffect that could be seen hours or even days after the original stimulation, suggesting the existence of long-term storage capability in the human visual system. Under usual experimental conditions, the aftereffect dissipates after a few seconds, but Masland was able to record apparent movement on a stationary spiral after $24 \mathrm{~h}$. The effect seems to indicate a long-term modification of the responsiveness of the nervous system and is limited to the part of the visual system stimulated by actual movement. The illusion of movement appears only after restimulation of these areas by a stationary stimulus. Masland's results suggested either that a new type of adaptation had been discovered, or that the effects observed were due to a learning process.

Hansel and Jones (1982), in a negative afterimage experiment, demonstrated that both short-term and long-term aftereffects may be evoked from the same retinal area and that the two effects are dependent on separate adaptation mechanisms. Evidence in support of the presence of twostage effects has been suggested in a number of contingent and noncontingent aftereffects experiments (Frome, Harris, \& Levinson, 1975; Hansel \& Mahmud, 1978). This presence is particularly evident in the case of movement aftereffets. In Masland's (1969) demonstration of the long-term movement aftereffect, transfer was not obtained from eye to eye, whereas in the case of the shortterm spiral aftereffect, such transfer is normally reported. These findings imply a central location for the effect, but suggest that there are two separate mechanisms underlying the effect-a long-term and a short-term mechanism.

The aim of Experiment 1 was to investigate the presence of the two separate movement aftereffects by modifying the experimental technique that was employed by Hansel

Address correspondence to the author at Department of Psychology, University of Dhaka, Dhaka-2, Bangladesh. and Jones (1982) for the investigation of the negative color afterimage (i.e., using positive followed by reverse negative adaptation sequences in relation to a particular retinal area). This technique was used to ensure that the original movement aftereffect was canceled and then reversed. The duration of the negative adaptation was obtained from a pilot test as being just sufficient to cancel and then reverse the original movement aftereffect. Successive tests at fixed intervals gave the original effect produced from the positive adaptation an opportunity to reappear.

\section{EXPERIMENT 1}

\section{Method}

Subjects. Twelve university students with normal or corrected-tonormal vision acted as subjects. All were naive with respect to the movement aftereffect.

Apparatus and Materials. The adapting stimulus was a 3.5-turn blackand-white arithmetic spiral. The black line subtended $0.69^{\circ}$ and the complete spiral $4.8^{\circ}$ of visual angle. Clockwise rotation of the spiral made it appear to contract.

The test stimulus was the same spiral presented in a stationary position.

Procedure. The subjects sat $2.4 \mathrm{~m}$ from the disc and watched the spiral. During adaptation, the spiral was rotated at $80 \mathrm{rpm}$ so that angular rate of expansion or contraction contours of the spiral was $0.91^{\circ}$ per second. Subjects were adapted for $10 \mathrm{~min}$ (positive adaptation) by fixating at the center of the spiral. Half of them were inducted to expanding spiral and the other half to contracting spiral. Following adaptation, subjects were tested with the same spiral in a stationary position exposed for $2 \mathrm{sec}$. The subject reported whether the spiral appeared to be contracting, expanding, or motionless. After a 5-min interval, the spiral was rotated in the reverse direction for 1 min (negative adaptation). The stationary spiral was then again presented for $2 \mathrm{sec}$ and the subject reported expansion, contraction, or lack of movement. The purpose of this test was to ensure that the initial movement aftereffect had been canceled. The test procedure was then given at 1-min intervals over a 5-min period, in order to give the original movement aftereffect an opportunity to reappear.

\section{Results}

After the positive adaptation, all subjects reported the movement in the direction reverse to that observed during adaptation. Each of them also reported the reversal 
of this original movement after the 1-min negative adaptation. Between the immediate test and the test after $5 \mathrm{~min}$, each subject's reports of movement gradually changed back to the original direction (Table 1).

\section{Discussion}

The results highlight the two separate mechanisms that appear to be present during testing: a long-term and a short-term adaptation mechanism. The long-term mechanism seems to build up relatively slowly; hence, it is persistent and is retained over long periods of time. The short-term effect, however, builds up fairly rapidly (in this instance, in $1 \mathrm{~min}$ ) and also disappears rapidly, displaced by the reappearance of the long-term effect. These results show a remarkable resemblance to those produced with color afterimages (Hansel \& Jones, 1982; Hansel \& Mahmud, 1978). This suggests that common principles underlie both effects, which are highlighted after similar experimental adaptation.

\section{EXPERIMENT 2}

To investigate more fully the apparent existence of two separate mechanisms underlying this effect, an additional 8 subjects were adapted following the same experimental procedure, but employing monocular adaptation. It was hypothesized that the initial long-term effect produced after positive adaptation would show interocular transfer, along with the subsequent short-term effect produced after negative adaptation. The recurrence of the original long-term effect, however, was expected to occur only in the eye that was adapted to movement.

\section{Method}

Subjects. Eight undergraduate students with normal or corrected-tonormal vision were enlisted as subjects.

Apparatus and Materials. The apparatus was identical to that employed in Experiment 1.

Procedure. With one eye of each subject occluded by an eye patch, 4 subjects adapted to an expanding spiral and 4 to a contracting spiral. The long-term adaptation time was $10 \mathrm{~min}$. The adaptation procedure was identical to that used in the previous experiment. Subjects were tested by fixating the stationary spiral, which was exposed for $2 \mathrm{sec}$. Each eye was tested separately. The unadapted eye was always tested first so that movement judgments were not biased by effects observed with the adapted eye.

\section{Results}

For all subjects after long-term adaptation, both the adapted and the occluded eye perceived the movement aftereffect in the appropriate direction, indicating that interocular transfer from the adapted to the unadapted eye

Table 1

Direction of Apparent Movement Reported by Subjects $(N=12)$ Viewing a Stationary Test Spiral

\begin{tabular}{lll}
\hline & \multicolumn{2}{c}{ Adaptation Spiral } \\
\cline { 2 - 3 } & Expanding Spiral & Contracting Spiral \\
\hline Positive adaptation to: & Expansion & Contraction \\
Observed aftereffect: & Contraction & Expansion \\
Negative adaptation to: & Contraction & Expansion \\
Immediate aftereffect: & Expansion & Contraction \\
Aftereffect 5 min later: & Contraction & Expansion \\
\hline
\end{tabular}

had taken place. Also after the 1-min neutralization adaptation, the unadapted eye as well as the adapted eye perceived a reversal of the original movement effect. During the 1-min successive tests, each subject reported a return to the original movement aftereffect in his/her adapted eye. In the occluded eye, however, no movement was reported in response to the stationary spiral, indicating that long-term adaptation persists only in the adapted eye.

\section{GENERAL DISCUSSION}

The results of these experiments show that two separate mechanisms are responsible for long-term and short-term adaptation to movement. The results are different from those with color, in that the short-term effect transfers from eye to eye, again indicating that the short-term effect arises at a later stage of processing than does the long-term effect.

For both movement and color, the long-term aftereffect rapidly decays and returns after the subject looks away and refixates the test pattern. This has been reported in a number of experiments, where it has been likened to a habituation effect operating in a learning process (Mayhew \& Anstis, 1972). It appears likely that, in the case of both color and movement aftereffects, the rapid decay during fixation of the longterm effect is due to the intrusion of the short-term adaptation process that temporarily removes the effects arising from the earlier long-term adaptation process.

Masland (1969) suggested that the results of his experiment reflect a "long-term modification of the responsiveness of the nervous system" (p. 821). The perception of visual movement is rarely a simple function of objective stimulus motion to a fixed retina. The neural activity of receptors early in the human visual system represents only an elementary level of visual processing. Hence, Masland suggested that one system is sensitive to motion per se, mediated by single cortical cells, and that a second responds to motion by encoding changes in perceived motion over perceived time. A dual mechanism is thought to be physiologically feasible in that vision is subserved jointly by phylogenetically old and new systems. Masland's (1969) suggestion also ties in with Exner's (1875) explanation that in the movement aftereffect, the sensation of pure movement is perceived irrespective of any movement stimulus.

Two or more separate adaptation processes are essential in a complex system for the perception of movement. In a highly complex system, it is quite feasible to expect separate adaptation processes to arise at many stages. The presence of separate stages of adaptation serve to balance or regulate the system. Adaptation often has to be very slow and gradual when the system balances to stability as a result of relatively permanent changes in the environment.

Long-term adaptation results in long-term stability. The short-term element, however, requires constant adjustments to rapidly changing features of the environment on a day-to-day basis (e.g., for a person to keep himself/herself upright and steady by making minor adjustments during movement). The constancy and stability of the visual world must take precedence over the perceived movement of individual parts of it.

The perception of movement cannot be described solely in terms of the function of individual motion-detection units. Motion-sensitive cortical cells must operate at an early level in the analysis of perceived movement in response to short-term fluctuations, such as the motion aftereffect. This is shown by the characteristics of the movement aftereffect (e.g., retinal specificity, lack of discrimination between test stimuli). It is suggested that the short-term movement aftereffect is due to neural adaptation at an early level in the analysis of movement. By prolonging adaptation to a moving stimulus, however, it is possible to demonstrate the presence of a long-term component, arising from more complex mechanisms higher up in the visual system, which determine the ultimate perception of objects in the real world. 


\section{REFERENCES}

ExNER, S. (1875). Ueber das Sehen Von Bewegungen Und die Theorie des Zusammengesetzen Auges, S. B. Akad, Wiss. Wien, 72, 156-190.

Frome, F., Harris, C. S., \& Hevinson, J. Z. (1975). Extremely longlasting shifts in perception of size after adaptation to gratings. Bulletin of the Psychonomic Society, 6, 433. (Abstract)

HANSEL, C. E. M., \& JoNES, R. A. (1982). Long-term retention of the negative afterimage. IRCS Medical Science, 10, 7-8.
Hansel, C. E. M., \& Mahmud, S. H. (1978). Comparable retention times for the negative color afterimage and the McCollough effect. Vision Research, 18, 1601-1605.

MASLAND, R. H. (1969). Visual motion perception experimental modification. Science, 165, 819-821.

MayheW, J. E. W., \& ANSTIS, S. M. (1972). Movement aftereffect contingent on color intensity and pattern. Perception \& Psychophysics, 12, 77-85.

(Manuscript received for publication February 27, 1987.) 